\title{
Changes in health-related quality of life of Chinese populations measured by the EQ- 5D-3 L: a comparison of the 2008 and 2013 National Health Services Surveys
}

\author{
Qiang Yao ${ }^{1,2} \mathbb{B}$, Chaojie Liu ${ }^{2} \mathbb{D}$, Yaoguang Zhang ${ }^{3 *}$ and Ling $X u^{4^{*}}$
}

\begin{abstract}
Backgrounds: The EuroQol Group Five-Dimensional (EQ-5D) instruments have been validated in China for measuring health-related quality of life (HRQoL) and are increasingly being used in health economic studies. However, there is paucity in the literature documenting long-term changes in the EQ-5D results in the Chinese populations. This study aims to identify such changes and their determinants using the EQ-5D-3 L instrument.

Methods: Data were obtained from the National Health Services Surveys in China, which included the EQ-5D-3 L since 2008. We compared the differences between the 2008 and 2013 surveys in the percentage of reported problems, visual analogue scale (VAS) scores, and the EQ-5D-3 L utility index derived from the national value sets. Factors associated with population changes in these EQ-5D results were identified using logistic, linear and Tobit regression models, respectively.
\end{abstract}

Results: Compared with 2008, reported problems in self-care (3.3\% vs 3.1\%), usual activities (4.8\% vs 4.6\%) and anxiety/depression (6.4\% vs 5.3\%) decreased, whereas reported problems in mobility (5.1\% vs $5.9 \%$ ) and pain/discomfort $(9.3 \%$ vs $12.6 \%)$ increased significantly $(p<0.05)$ in 2013 . The regression models revealed a rise $(\beta=1.61, p<0.001)$ in VAS scores, but a slight drop $(\beta=-0.01, p<0.001)$ in utility index in 2013 compared with 2008 after controlling for variations in demographic, behavioral, socioeconomic and residential variables. But the effect sizes of the changes over time (estimated by "average change divided by baseline standard deviation") did not reach the threshold of clinical importance after adjustment for variations in other factors. Higher socioeconomic status (in terms of education, income and residential location) was associated with better EQ-5D-3 $L$ results.

Conclusion: The changing trend (decrease) of the utility index is contradictory to that (increase) of the VAS scores, although neither is deemed clinically important. It is evident that socioeconomic and regional disparities in HRQoL exist in China.

Keywords: Health-related quality of life, EQ-5D-3 L, National Health Services Surveys, China

\footnotetext{
* Correspondence: zhangyg@nhfpc.gov.cn; xuling@nhfpc.gov.cn

${ }^{3}$ Center for Health Statistics and Information, National Health Commission,

Beijing, China

${ }^{4}$ Health Human Resources Development Center, National Health

Commission, Beijing, China

Full list of author information is available at the end of the article
}

(c) The Author(s). 2019 Open Access This article is distributed under the terms of the Creative Commons Attribution 4.0 International License (http://creativecommons.org/licenses/by/4.0/), which permits unrestricted use, distribution, and reproduction in any medium, provided you give appropriate credit to the original author(s) and the source, provide a link to the Creative Commons license, and indicate if changes were made. The Creative Commons Public Domain Dedication waiver (http://creativecommons.org/publicdomain/zero/1.0/) applies to the data made available in this article, unless otherwise stated. 


\section{Introduction}

Health-related quality of life (HRQoL) is a multi-dimensional concept that measures self-reported well-being in physical, mental, and social functioning. It captures the impacts of health problems and diseases on quality of life [1-4]. HRQoL has been widely used for assessing outcomes of medical interventions as well as serving as a population health indicator, guiding health policy development and resources allocation $[5,6]$. It puts a quality perspective into the measurement of years of life [7, 8]. In combination with traditional health indicators such as mortality and morbidity, a comprehensive indicator (e.g. quality-adjusted life years or QALYs) can be calculated to inform clinical, funding, public policy and management decisions on health products and technologies [9-13].

Over the past few decades, significant progress has been made in measuring HRQoL. The EuroQol Group Five-Dimensional (EQ-5D) series is perhaps one of the most simple and frequently used instruments since early 1990s [14]. The EQ-5D consists of a descriptive system and a visual analogue scale (VAS). The descriptive system comprises five dimensions. Problems related to these dimensions were measured either at three levels (EQ-5D-3 L) or five levels (EQ-5D-5 L) [14]. The combination of reported problems can be converted into a single summary utility index by applying the value sets based on public preferences. The EQ-5D has been recommended by the National Institute for Health and Care Excellence in the UK [8] and the 2011 Guidelines for Pharmacoeconomic Evaluations in China [15].

The EQ-5D-3 L instrument has been validated in Chinese populations [16, 17] and is increasingly being used for assessing HRQoL in the general population [18-20], people with special demographic characteristics [21-25], and people living with chronic conditions $[4,26-34]$. Approaches to studies in China using the EQ-5D-3 L evolved over time. The first stage (before 2014) is featured with studies applying the value sets developed in the UK and Japan due to a lack of Chinese value sets [22, 35]. Researchers soon noticed significant differences in public preferences on health status that would result in varied EQ-5D-3 L value sets across nations [36, 37]. In 2014, Liu GG et al. [15] published the EQ-5D-3 L value sets derived from a sample of urban Chinese residents, triggering a wave of second stage studies [20, 38-40]. The localised value sets generated significant different EQ-5D-3 L utility results compared with those derived from the UK and Japanese populations [41]. Unfortunately, the EQ-5D-3 L value sets developed by Liu GG suffer from some major limitations due to the biased sampling: participants were selected conveniently from a few big cities. The authors themselves suggested a need for further refinement of the EQ-5D-3 L value sets in China based on a more inclusive and representative sample [15]. Indeed, there exist great socioeconomic and health disparities between urban and rural populations in China [19]. Public preferences on health status also vary between urban (especially those living in big cities) and rural residents [42]. Such an urban-rural difference in public preferences on health remains even after controlling for variations in socioeconomic status [18]. This highlights the importance of the development of the national representative EQ-5D-3 L value sets in China [15, 43]. In 2018, the national value sets derived from a large representative sample were eventually made available in China [15, 43].

This study aimed to identify changes in the EQ-5D-3 L results over time in China and their determinants. The study was undertaken using the EQ-5D-3 L instead of the EQ-5D-5 L for two reasons. First, two surveys five years apart (2008 and 2013) were available. Over this period of time, China experienced dramatic changes in social and economic development, including reforms of the health system. In this context, changes in HRQoL are likely to occur. Second, the EQ-5D-3 L generates a utility index based on the national value sets derived from public preferences, as well as a VAS indicating individual ratings on their own conditions [44-46]. This enables us to compare changing trends reflected by the two indicators and identify the determinants of contradictions, if any exist.

\section{Data and methods}

\section{Study design and data collection \\ Data source and sampling method}

Data were obtained from the 2008 and 2013 National Health Services Surveys (NHSS), the largest national wide health surveys in China. The NHSS have been conducted every five years since 1993 with coordination from the Centre for Health Statistics and Information under the Ministry of Health [47]. The EQ-5D-3 L has been validated in various populations in China [16-18, 28-34, 48] and incorporated into the NHSS since 2008 [19].

A four-stage stratified cluster probability sampling strategy was adopted to select representative participants in the NHSS $[49,50]$. Each stage involved a systematic random sampling approach. In the first stage, urban cities and rural counties (94 in 2008 and 156 in 2013) were selected from the 31 provinces in mainland China in proportion to their population size. In the second stage, five sub-districts/townships in each selected city/county were identified. The third stage narrowed the sample down to two residential communities/villages in each district/township. Finally, 60 households from each residential community/village were invited to participate in the NHSS. All family members in a sampled household 
were eligible to participate in the NHSS. However, the EQ-5D-3 L was only administered to those who were 15 years or older. This resulted in a final sample size of 120,709 individual respondents in 2008 (excluding 24,921 questionnaires with a proxy respondent and 601 returned questionnaires containing missing values in the EQ-5D-3 L) and 188,720 individual respondents in 2013 (excluding 41,148 questionnaires with a proxy respondent and 196 returned questionnaires containing missing values in the EQ-5D-3 L) for data analyses in this study. The questionnaires with a proxy respondent were excluded because their VAS ratings were unlikely reliable.

\section{Questionnaire survey}

The questionnaires were administered through face-toface household interviews conducted by trained local medical workers. The interviewers were rigorously selected considering their qualifications, professional knowledge, sense of responsibility, attitudes, and communication skills. Pre-survey training workshops were offered to all of the interviewers following a standardised protocol. Eligible interviewers had to demonstrate their proper understanding about the purpose of the NHSS and ability to meet data collection standards developed by the Centre for Health Statistics and Information. They also needed to follow pre-defined instructions when problems arose during data collection [49-51].

The face-to-face interviews occurred in the households of participants. Prior to the interviews, the interviewers explained the purpose and procedure of the survey and obtained oral informed consent from each participant. Family members were allowed to serve as a proxy respondent only when the selected participant was younger than 15 years, absent from home, or unable to communicate at the time of the survey. Overall, less than $18 \%$ of the returned questionnaire (17\% in 2008 and $18 \%$ in 2013) were completed by a proxy respondent $[50,52]$.

A quality assurance team was established in each participating sub-district/township. The returned questionnaires were checked by the quality assurance officers for completeness at the end of each day. Missing data, if any, were addressed through a second interview by the same interviewers. The sampled households were not allowed to be replaced unless three interview attempts failed or the sample size of the residential community/village did not reach 60 [19]. The survey supervisors in each city/county re-interviewed $5 \%$ of their local participating households for selected key questions (eight in 2008 and fourteen in 2013) through telephone interviews or field visits. The repeated interviews showed over $95 \%$ of consistency with the original ones $[49,52]$. The representativeness of the sample was evident in terms of Myer's Index, DELTA dissimilarity coefficient and GINI concentration ratio for age structure and household size $[49,50,52,53]$.

\section{Measurements Dependent variables}

Three indicators were calculated: percentage of reported problems, utility index and VAS scores.

The descriptive system of EQ-5D comprises five dimensions: mobility (MO), self-care (SC), usual activities (UA), pain/discomfort (PD) and anxiety/depression (AD). This study used the EQ-5D-3 L, measuring problems at three levels: no problem, moderate problem, severe problem.

The combination of reported problems on the five dimensions for each individual was converted into a summarised utility index $(U)$. The conversion was based on the value sets developed by Zhuo et al. [43]. It assigns a value to each of the 243 health states classified by the EQ-5D-3 L:

$$
\begin{aligned}
U=1- & 0.0766^{*} M O 2+0.2668^{*} M O 3+0.0441^{*} S C 2 \\
& +0.2912^{*} S C 3+0.0370^{*} U A 2+0.0538^{*} U A 3 \\
& +0.0274^{*} P D 2+0.0409^{*} P D 3+0.0359^{*} A D 2 \\
& \left.+0.1771^{*} A D 3\right)
\end{aligned}
$$

where $\mathrm{MO} 2, \mathrm{SC} 2, \mathrm{UA} 2, \mathrm{PD} 2$ and $\mathrm{AD} 2$ were given a value of 1 if a "moderate problem" presented in the respective dimension, or 0 otherwise. Similarly, MO3, SC3, UA3, PD3 and AD3 were given a value of 1 if an "extreme problem" presented in the respective dimension, or 0 otherwise. This resulted in a possible utility index ranging from 0.1702 to 1.0000 [43].

There exist two value sets in China for the EQ-5D-3 L derived from the Time Trade-Off (TTO) technique: one developed by Liu et al. and another by Zhuo et al. $[15,43]$. Both value sets showed smaller disutility for the PD dimension than that from some other countries such as the UK. However, in both cases, linear correlations between the disutility values and the seriousness of health problems remained evident. Some researchers argued that Chinese people, in particular those living in rural areas, are more tolerant to pain problems than the western populations, which may result in smaller disutility for the PD dimension in China [54]. In this study, we chose to adopt the value sets developed by Zhuo and colleagues for several reasons. Firstly, there exist some differences between Liu's and Zhuo's value sets. Secondly, Liu's value sets failed to consider the preference of rural residents in China. But urban-rural disparities in health, social and economic development were still large in China. Finally, Zhuo's value sets were derived from a nationally representative sample.

The EQ-5D-3 L also contains a VAS, asking respondents to rate their overall health on a scale ranging from 'worst imaginable health state' (0) to 'best imaginable 
health state' (100). VAS reflects individual ratings on their own health $[18,19]$.

\section{Independent variables}

Changes in HRQoL are determined by a variety of factors at the individual, family, community and society levels [55-57]. There exist several models describing the social and ecological determinants of health [58-62]. In this study, we used the widely cited Dahlgren-Whitehead rainbow model to guide the selection of independent variables. The NHSS covered five broad categories of determinants of health $[19,63]$ :

Demographic variables: Gender and age represent the basic biological feature of respondents.

Behavioral variables: The NHSS collected data regarding the current status of smoking (past week), drinking (past 12 months), and physical exercise (past 6 months) of the respondents. Respondents were asked whether and how frequent they had engaged in the aforementioned behaviors at the time of the survey. Respondents were deemed to be physically active if they engaged in regular physical activities at least once a week over the past 6 months.

Socioeconomic variables: Socioeconomic status was measured by educational attainment and household income. Respondents were asked to indicate the highest level of school education they had attained. Household income was estimated based on self-reported average income per capita and converted into quintiles (from the lowest 20\% to the highest 20\%).

Residential variables: Respondents were divided into two groups, urban or rural, based on their geographical location in three regions: eastern, central and western. There exist great geographic disparities in socioeconomic development in China. Urban residents enjoy a higher level of entitlement than their rural counterparts. Overall, the eastern coastal region of China is highly developed, whereas the western region lags far behind, with the central region being somewhere in between.

Time variable: China's most recent health system reform started in 2009. The reform achieved great progress in a few years in terms of universal coverage of health insurance and improved access to medical care $[49,64]$. We expected that the two rounds of NHSS would capture potential changes resulting from the reform.

\section{Statistical analyses}

We presented the percentages of reported problems on the five dimensions of EQ-5D-3 L. Reported "extreme problems" were rare, and thus were merged with the category of "moderate problems" [38, 47]. Differences in the percentages of reported problems were tested using $x^{2}$ tests. Logistic regression models were established to determine the changes over the two years after controlling for the influence of other independent variables. A $p$ value of less than 0.01 was considered statistically significant in line with the Bonferroni adjustment method for multiple comparisons [65].

Means of the utility index and VAS scores were calculated and compared using student $t$ tests or analysis of variance (ANOVA). Linear regression models were established to determine changes in VAS scores over the two years after controlling for variations in other independent variables. Because the utility index is bounded to 1 , Tobit regression models were established to determine changes in the utility index after controlling for variations in other independent variables [4]. A $p$ value of 0.05 was considered statistically significant in the linear and Tobit regression analyses.

The importance of changes in the utility index and VAS scores were also estimated using the indicator of effect size: average change divided by baseline standard deviation (SD), which was proposed by Cohen [66]. The Cohen effect size has now been widely used in identifying the importance of changes: 0.2 small; 0.5 medium; 0.8 large. A medium effect size is usually considered as a difference with clinical meaning [67]. In this study, the medium effect size (0.5) was considered as a threshold for minimal importance of changes in the utility index and VAS scores.

All statistical analyses were performed using STATA 14.0 for Windows.

\section{Results}

\section{Characteristics of respondents}

Slightly less than half of the respondents were male (47.83\%). Elderly respondents accounted for less than $20 \%$ of respondents (16.45\%). More than half of the respondents resided in rural areas (58.79\%). Compared with 2008, the 2013 respondents were older $(p<0.001)$, more likely to smoke $(p<0.05)$, drink $(p<0.001)$ and exercise $(p<0.001)$; they also reported higher levels of education $(p<0.001)$. There was a dramatic increase $(p<0.001)$ in the proportion of urban respondents: $49.84 \%$ in 2013 compared with $27.71 \%$ in 2008 (Table 1).

\section{Changes in health-related quality of life Reported health problems}

Pain/discomfort was the most frequently reported problem (11.3\%), followed by anxiety/depression (5.7\%) and mobility (5.6\%). Problems in self-care were least reported (3.1\%). Compared with 2008, reported problems increased in pain/discomfort (by 3.3 percentage points) and mobility (by 0.7 percentage points) in 2013. By contrast, reported problems in anxiety/depression, self-care and usual activities decreased by 1.2, 0.2 and 0.2 percentage points, respectively (Table 2 ). 
Table 1 Characteristics of respondents

\begin{tabular}{|c|c|c|c|c|c|c|c|c|}
\hline \multirow[t]{2}{*}{ Characteristics } & \multicolumn{2}{|l|}{2008} & \multicolumn{2}{|l|}{2013} & \multicolumn{2}{|l|}{ Total } & \multirow[t]{2}{*}{$x^{2}$} & \multirow{2}{*}{$\begin{array}{l}P \\
\text { value }\end{array}$} \\
\hline & $n$ & $\%$ & $n$ & $\%$ & $n$ & $\%$ & & \\
\hline \multicolumn{9}{|l|}{ Demographic variables } \\
\hline \multicolumn{9}{|l|}{ Gender } \\
\hline Male & 58,169 & 48.19 & 89,830 & 47.60 & 147,999 & 47.83 & 10.26 & 0.001 \\
\hline Female & 62,540 & 51.81 & 98,890 & 52.40 & 161,430 & 52.17 & & \\
\hline \multicolumn{9}{|l|}{ Age } \\
\hline $15 \sim 44$ & 58,233 & 48.24 & 73,522 & 38.96 & 131,755 & 42.58 & 2600.00 & $<0.001$ \\
\hline $45 \sim 64$ & 45,252 & 37.49 & 81,532 & 43.20 & 126,784 & 40.97 & & \\
\hline $65 \sim$ & 17,224 & 14.27 & 33,666 & 17.84 & 50,890 & 16.45 & & \\
\hline \multicolumn{9}{|l|}{ Behavioral variables } \\
\hline \multicolumn{9}{|l|}{ Smoking } \\
\hline Yes & 30,925 & 25.73 & 49,208 & 26.09 & 80,133 & 25.95 & 5.07 & 0.024 \\
\hline No & 89,285 & 74.27 & 139,399 & 73.91 & 228,684 & 74.05 & & \\
\hline \multicolumn{9}{|l|}{ Drinking } \\
\hline Yes & 15,485 & 13.04 & 44,005 & 23.32 & 59,490 & 19.35 & 4900.00 & $<0.001$ \\
\hline No & 103,229 & 86.96 & 144,706 & 76.68 & 247,935 & 80.65 & & \\
\hline \multicolumn{9}{|l|}{ Regular physical activity } \\
\hline Yes & 26,831 & 22.60 & 55,843 & 29.67 & 82,674 & 26.93 & 1800.00 & $<0.001$ \\
\hline No & 91,896 & 77.40 & 132,372 & 70.33 & 224,268 & 73.07 & & \\
\hline \multicolumn{9}{|l|}{ Socioeconomic variables } \\
\hline \multicolumn{9}{|l|}{ Educational attainment } \\
\hline Illiterate & 18,841 & 15.62 & 22,709 & 12.03 & 41,550 & 13.43 & 2500.00 & $<0.001$ \\
\hline Primary school & 33,630 & 27.88 & 48,953 & 25.94 & 82,583 & 26.70 & & \\
\hline Junior middle school & 43,042 & 35.69 & 65,877 & 34.91 & 108,919 & 35.21 & & \\
\hline Senior middle school & 17,941 & 14.87 & 32,435 & 17.19 & 50,376 & 16.29 & & \\
\hline University/college & 7160 & 5.94 & 18,746 & 9.93 & 25,906 & 8.37 & & \\
\hline \multicolumn{9}{|c|}{ Household income ranking in local } \\
\hline Lowest $(<20 \%)$ & 22,523 & 18.68 & 35,702 & 18.93 & 58,225 & 18.83 & 39.67 & $<0.001$ \\
\hline $20 \%-$ & 23,369 & 19.38 & 35,471 & 18.80 & 58,840 & 19.03 & & \\
\hline $40 \%-$ & 24,277 & 20.13 & 37,124 & 19.68 & 61,401 & 19.86 & & \\
\hline $60 \%-$ & 24,166 & 20.04 & 39,084 & 20.72 & 63,250 & 20.45 & & \\
\hline Highest ( $\geq 80 \%$ ) & 26,256 & 21.77 & 41,251 & 21.87 & 67,507 & 21.83 & & \\
\hline \multicolumn{9}{|l|}{ Residential variables } \\
\hline \multicolumn{9}{|l|}{ Residency } \\
\hline Rural & 87,262 & 72.29 & 94,656 & 50.16 & 181,918 & 58.79 & $15,000.00$ & $<0.001$ \\
\hline Urban & 33,447 & 27.71 & 94,064 & 49.84 & 127,511 & 41.21 & & \\
\hline \multicolumn{9}{|l|}{ Region } \\
\hline Eastern & 42,305 & 35.05 & 66,575 & 35.28 & 108,880 & 35.83 & 1200.00 & $<0.001$ \\
\hline Central & 33,175 & 27.48 & 58,306 & 30.90 & 91,481 & 30.10 & & \\
\hline Western & 45,229 & 37.47 & 63,839 & 33.83 & 103,535 & 34.07 & & \\
\hline Total & 120,709 & 100.00 & 188,720 & 100.00 & 309,429 & 100.00 & & \\
\hline
\end{tabular}


Table 2 Reported health problems of respondents

\begin{tabular}{|c|c|c|c|c|c|c|c|c|}
\hline \multirow[t]{2}{*}{ EQ-5D Dimensions } & \multicolumn{2}{|l|}{2008} & \multicolumn{2}{|l|}{2013} & \multicolumn{2}{|l|}{ Total } & \multirow[t]{2}{*}{$x^{2}$} & \multirow[t]{2}{*}{$p$} \\
\hline & $n$ & $\%$ & $n$ & $\%$ & $n$ & $\%$ & & \\
\hline \multicolumn{9}{|l|}{ Mobility } \\
\hline No problem & 114,502 & 94.9 & 177,667 & 94.1 & 292,169 & 94.4 & 71.40 & $<0.001$ \\
\hline Moderate/ Extreme problem & 6207 & 5.1 & 11,053 & 5.9 & 17,260 & 5.6 & & \\
\hline \multicolumn{9}{|l|}{ Self-care } \\
\hline No problem & 116,774 & 96.7 & 182,955 & 97.0 & 299,729 & 96.9 & 10.20 & 0.001 \\
\hline Moderate/ Extreme problem & 3935 & 3.3 & 5765 & 3.1 & 9700 & 3.1 & & \\
\hline \multicolumn{9}{|l|}{ Usual activities } \\
\hline No problem & 114,881 & 95.2 & 179,966 & 95.4 & 294,847 & 95.3 & 5.89 & 0.015 \\
\hline Moderate/ Extreme problem & 5828 & 4.8 & 8754 & 4.6 & 14,582 & 4.7 & & \\
\hline \multicolumn{9}{|l|}{ Pain/discomfort } \\
\hline No problem & 109,548 & 90.8 & 164,920 & 87.4 & 274,468 & 88.7 & 831.82 & $<0.001$ \\
\hline Moderate/ Extreme problem & 11,161 & 9.3 & 23,800 & 12.6 & 34,961 & 11.3 & & \\
\hline \multicolumn{9}{|l|}{ Anxiety/depression } \\
\hline No problem & 112,955 & 93.6 & 178,767 & 94.7 & 291,722 & 94.3 & 180.39 & $<0.001$ \\
\hline Moderate/ Extreme problem & 7754 & 6.4 & 9953 & 5.3 & 17,707 & 5.7 & & \\
\hline
\end{tabular}

Bold figures indicate a significant increase in 2013 compared with those in $2008(p<0.01)$

\section{Utility index and VAS scores}

Respondents who were male, younger, richer and had higher educational attainments reported lower health problems than the others, resulting in a higher utility index and VAS scores. Smoking, drinking and regular exercise were associated with less reported health problems and a higher utility index and VAS scores. Compared with rural respondents, urban respondents reported less health problems and consequently had a higher utility index, but they rated lower in VAS (Table 3).

Overall, the respondents had an average utility index around 0.985 and an average VAS score around 80. Compared with 2008, there was a significant decrease $(p<0.001)$ in the utility index, but an increase $(p<0.001)$ in VAS scores in 2013, albert in very small effect sizes (- 0.01 and 0.06 , respectively). However, some subgroups stratified by age and region defied the overall trends, showing an increase in both the utility index and VAS scores (Table 3). Nonetheless, neither changes in the utility index (effect sizes ranging from -0.31 to 0.03 ) nor changes in VAS scores (effect sizes ranging from -0.01 to 0.17 ) reached the threshold of minimal clinical importance (Table 3).

\section{Findings of multivariate regression models}

Changes in reported health problems, the utility index and VAS scores over time remained significant after controlling for the influence of other independent variables.

\section{Factors associated with reported health problems}

The logistic regression models confirmed that there was an increased likelihood of reporting problems in 2013 in pain/discomfort $(A O R=1.34$ ) and mobility (AOR $=1.07$, but a decreased likelihood of reporting problems in self-care $(\mathrm{AOR}=0.90)$, usual activity $(\mathrm{AOR}=0.92)$, and anxiety/depression $(\mathrm{AOR}=0.80)$ compared with 2008 (Table 4).

\section{Factors associated with the utility index and VAS scores}

The multiple linear and Tobit regression models confirmed the contradictory changing trends over time: decreased $(\beta=-0.01, p<0.001)$ utility index vs increased $(\beta=1.61, \mathrm{p}<0.001)$ VAS scores, after adjustment for variations in the other independent variables. But the effect sizes of those changes over time did not reach the threshold of minimal clinical importance (Table 5). Age, education, income and residential location were significant predictors of the utility index and VAS scores. Their effect sizes reached the threshold of minimal clinical importance for the utility index. But for VAS scores, only the age effect reached the threshold of minimal clinical importance.

\section{Discussion}

Changes in HRQoL over time in the Chinese populations between 2008 and 2013 are minimal, if ever exist, according to this study. Compared with 2008, reported problems in mobility and pain/discomfort increased in 2013, but reported problems in anxiety/depression, 
Table 3 Reported problems, health utility index and VAS scores stratified by independent variables

\begin{tabular}{|c|c|c|c|c|c|c|c|c|c|c|c|c|c|c|c|c|c|c|}
\hline \multirow[t]{2}{*}{ Variables } & \multicolumn{2}{|c|}{ Mobility } & \multicolumn{2}{|c|}{ Self-care } & \multicolumn{2}{|c|}{$\begin{array}{l}\text { Usual } \\
\text { activities }\end{array}$} & \multicolumn{2}{|c|}{$\begin{array}{l}\text { Pain/ } \\
\text { discomfort }\end{array}$} & \multicolumn{2}{|c|}{$\begin{array}{l}\text { Anxiety/ } \\
\text { depression }\end{array}$} & \multicolumn{4}{|c|}{ Mean Utility index (SD) } & \multicolumn{4}{|c|}{ Mean VAS scores (SD) } \\
\hline & 2008 & 2013 & 2008 & 2013 & 2008 & 2013 & 2008 & 2013 & 2008 & 2013 & 2008 & & 2013 & & 2008 & & 2013 & \\
\hline \multicolumn{19}{|l|}{ Gender } \\
\hline male & 4.9 & 5.7 & 3.1 & 3.0 & 4.5 & 4.5 & 8.0 & 11.0 & 5.7 & 4.6 & 0.986 & $(0.058)$ & 0.986 & $(0.056)$ & 80.9 & $(13.8)$ & 81.6 & (13.5) \\
\hline female & 5.4 & 6.0 & 3.5 & 3.1 & 5.1 & 4.8 & 10.4 & 14.1 & 7.1 & 5.9 & 0.985 & $(0.059)$ & 0.984 & $(0.056)$ & 79.4 & $(14.2)$ & 80.3 & (13.9) \\
\hline \multicolumn{19}{|l|}{ Age } \\
\hline $15 \sim 44$ & 1.3 & 1.0 & 0.8 & 0.6 & 1.2 & 0.9 & 3.1 & 3.7 & 3.1 & 2.3 & 0.996 & $(0.029)$ & 0.986 & $(0.029)$ & 85.4 & $(11.3)$ & 86.9 & $(10.4)$ \\
\hline $45 \sim 64$ & 5.1 & 4.8 & 3.0 & 2.3 & 4.6 & 3.6 & 11.4 & 14.0 & 7.5 & 5.8 & 0.985 & $(0.057)$ & 0.986 & $(0.050)$ & 77.5 & $(13.7)$ & 79.3 & (13.3) \\
\hline $65 \sim$ & 18.5 & 19.0 & 12.5 & 10.4 & 17.5 & 15.3 & 24.2 & 28.7 & 14.7 & 10.5 & 0.953 & $(0.105)$ & 0.956 & $(0.099)$ & 69.2 & $(15.0)$ & 71.7 & (14.9) \\
\hline \multicolumn{19}{|l|}{ Smoking status } \\
\hline Yes & 4.2 & 4.7 & 2.4 & 2.2 & 3.8 & 3.5 & 8.2 & 10.8 & 5.8 & 4.5 & 0.988 & $(0.049)$ & 0.988 & $(0.045)$ & 80.6 & $(13.4)$ & 81.6 & (13.0) \\
\hline No & 5.5 & 6.3 & 3.5 & 3.4 & 5.2 & 5.1 & 9.6 & 13.2 & 6.7 & 5.5 & 0.984 & $(0.061)$ & 0.984 & $(0.060)$ & 80.0 & $(14.2)$ & 80.7 & (14.0) \\
\hline \multicolumn{19}{|l|}{ Drinking status } \\
\hline Yes & 3.3 & 3.8 & 1.7 & 1.5 & 2.6 & 2.5 & 7.3 & 10.3 & 4.3 & 4.1 & 0.991 & $(0.039)$ & 0.991 & $(0.037)$ & 81.2 & $(12.8)$ & 82.3 & $(12.5)$ \\
\hline No & 5.4 & 6.5 & 3.5 & 3.5 & 5.2 & 5.3 & 9.6 & 13.3 & 6.8 & 5.6 & 0.985 & $(0.061)$ & 0.983 & $(0.061)$ & 79.9 & $(14.2)$ & 80.5 & (14.1) \\
\hline \multicolumn{19}{|l|}{ Physical activity } \\
\hline Yes & 4.0 & 4.5 & 2.1 & 2.0 & 3.4 & 3.2 & 7.6 & 11.9 & 4.4 & 4.3 & 0.990 & $(0.040)$ & 0.989 & $(0.038)$ & 80.3 & $(13.8)$ & 80.9 & (13.1) \\
\hline No & 5.5 & 6.4 & 3.6 & 3.5 & 5.2 & 5.3 & 9.6 & 12.9 & 6.9 & 5.7 & 0.984 & $(0.063)$ & 0.983 & $(0.062)$ & 80.1 & $(14.1)$ & 80.9 & $(14.0)$ \\
\hline \multicolumn{19}{|l|}{ Education status } \\
\hline Illiterate & 13.2 & 15.4 & 9.0 & 8.9 & 13.0 & 13.4 & 20.6 & 26.6 & 14.6 & 11.7 & 0.964 & $(0.090)$ & 0.961 & $(0.091)$ & 72.4 & $(15.5)$ & 73.1 & (15.4) \\
\hline Primary school & 6.0 & 8.0 & 3.8 & 4.0 & 5.7 & 6.1 & 11.5 & 17.1 & 7.5 & 6.6 & 0.983 & $(0.064)$ & 0.980 & $(0.063)$ & 78.1 & $(14.1)$ & 78.2 & (14.1) \\
\hline $\begin{array}{l}\text { Junior middle } \\
\text { school }\end{array}$ & 2.6 & 3.6 & 1.5 & 1.8 & 2.3 & 2.8 & 5.3 & 9.0 & 4.0 & 3.9 & 0.992 & $(0.041)$ & 0.990 & $(0.044)$ & 83.2 & $(12.5)$ & 83.1 & (12.6) \\
\hline $\begin{array}{l}\text { Senior middle } \\
\text { school }\end{array}$ & 2.6 & 2.9 & 1.4 & 1.4 & 2.1 & 2.2 & 4.8 & 7.9 & 3.3 & 3.2 & 0.993 & $(0.040)$ & 0.992 & $(0.041)$ & 83.2 & $(12.7)$ & 83.4 & (12.5) \\
\hline $\begin{array}{l}\text { University/college } \\
\text { or above }\end{array}$ & 1.9 & 1.7 & 1.0 & 0.8 & 1.5 & 1.2 & 3.7 & 5.0 & 2.3 & 2.5 & 0.995 & $(0.031)$ & 0.995 & $(0.028)$ & 83.6 & $(12.3)$ & 85.4 & $(11.2)$ \\
\hline \multicolumn{19}{|l|}{ Income level } \\
\hline Lowest (< 20\%) & 8.0 & 9.8 & 5.2 & 5.3 & 7.7 & 8.1 & 12.5 & 18.3 & 9.9 & 10.0 & 0.977 & $(0.074)$ & 0.975 & $(0.072)$ & 77.0 & $(15.5)$ & 77.7 & (15.6) \\
\hline $20 \%-$ & 5.2 & 6.1 & 3.3 & 3.2 & 4.8 & 4.8 & 9.5 & 13.0 & 6.8 & 5.7 & 0.985 & $(0.060)$ & 0.984 & $(0.058)$ & 79.8 & $(14.1)$ & 80.5 & (13.9) \\
\hline $40 \%-$ & 4.9 & 5.0 & 3.0 & 2.6 & 4.4 & 3.9 & 8.7 & 11.7 & 6.0 & 4.6 & 0.987 & $(0.055)$ & 0.987 & $(0.052)$ & 80.5 & (13.8) & 81.6 & (13.3) \\
\hline $60 \%-$ & 4.0 & 4.6 & 2.5 & 2.3 & 3.9 & 3.5 & 7.8 & 10.7 & 5.0 & 4.2 & 0.989 & $(0.051)$ & 0.988 & $(0.049)$ & 81.2 & $(13.4)$ & 81.9 & (12.9) \\
\hline Highest ( $\geq 80 \%)$ & 3.9 & 4.3 & 2.6 & 2.2 & 3.7 & 3.3 & 8.0 & 10.0 & 5.0 & 3.8 & 0.989 & $(0.049)$ & 0.989 & $(0.047)$ & 81.7 & $(13.1)$ & 82.4 & (12.6) \\
\hline \multicolumn{19}{|l|}{ Residency } \\
\hline Urban & 4.7 & 5.7 & 2.8 & 2.8 & 4.8 & 4.3 & 9.3 & 12.5 & 6.4 & 5.0 & 0.987 & $(0.055)$ & 0.985 & $(0.055)$ & 79.3 & $(14.0)$ & 80.6 & (13.8) \\
\hline Rural & 5.3 & 6.0 & 3.5 & 3.3 & 5.2 & 5.0 & 9.8 & 12.8 & 7.0 & 5.6 & 0.985 & $(0.060)$ & 0.984 & $(0.058)$ & 80.4 & $(14.1)$ & 81.2 & (13.7) \\
\hline \multicolumn{19}{|l|}{ Region } \\
\hline Eastern & 4.4 & 5.4 & 2.7 & 2.8 & 4.0 & 4.2 & 8.2 & 11.1 & 4.7 & 4.2 & 0.988 & $(0.053)$ & 0.986 & $(0.054)$ & 81.7 & (13.6) & 82.1 & $(13.2)$ \\
\hline Central & 5.1 & 6.0 & 3.3 & 3.1 & 4.8 & 4.6 & 8.9 & 13.4 & 5.1 & 5.5 & 0.986 & $(0.061)$ & 0.984 & $(0.058)$ & 79.9 & $(14.0)$ & 80.6 & (14.0) \\
\hline Western & 5.9 & 6.2 & 3.7 & 3.2 & 5.6 & 5.1 & 10.5 & 13.5 & 9.0 & 5.5 & 0.983 & $(0.061)$ & 0.984 & $(0.057)$ & 78.8 & $(14.4)$ & 79.9 & (13.9) \\
\hline Total & 5.1 & 5.9 & 3.3 & 3.1 & 4.8 & 4.6 & 9.3 & 12.6 & 6.4 & 5.3 & 0.985 & $(0.058)$ & 0.985 & $(0.056)$ & 80.1 & $(14.1)$ & 80.9 & (13.7) \\
\hline
\end{tabular}


Table 4 Multiple logistic regression analyses on reported health problems

\begin{tabular}{|c|c|c|c|c|c|c|c|c|c|c|c|c|c|c|c|}
\hline \multirow[t]{2}{*}{ Independent variable } & \multicolumn{3}{|l|}{ Mobility } & \multicolumn{3}{|l|}{ Self-care } & \multicolumn{3}{|c|}{ Usual activity } & \multicolumn{3}{|c|}{ Pain/discomfort } & \multicolumn{3}{|c|}{ Anxiety/depression } \\
\hline & AOR & \multicolumn{2}{|l|}{$95 \% \mathrm{Cl}$} & $\mathrm{AOR}$ & \multicolumn{2}{|l|}{$95 \% \mathrm{Cl}$} & $\mathrm{AOR}$ & \multicolumn{2}{|l|}{$95 \% \mathrm{Cl}$} & AOR & \multicolumn{2}{|c|}{$95 \% \mathrm{Cl}$} & $\mathrm{AOR}$ & \multicolumn{2}{|c|}{$95 \% \mathrm{Cl}$} \\
\hline \multicolumn{16}{|l|}{ Gender (Male as reference) } \\
\hline Female & $0.77^{* *}$ & 0.74 & 0.80 & $0.69^{* *}$ & 0.65 & 0.72 & $0.71^{* *}$ & 0.68 & 0.74 & $1.18^{* *}$ & 1.14 & 1.21 & $1.05^{*}$ & 1.01 & 1.10 \\
\hline \multicolumn{16}{|l|}{ Age ( $<45$ years as reference) } \\
\hline $45 \sim 64$ & $4.14^{* *}$ & 3.90 & 4.40 & $3.57^{* *}$ & 3.30 & 3.87 & $3.62^{* *}$ & 3.39 & 3.86 & $3.71^{* *}$ & 3.58 & 3.85 & $2.37^{* *}$ & 2.27 & 2.48 \\
\hline $65 \sim$ & $15.95^{* *}$ & 14.97 & 17.00 & $14.49^{* *}$ & 13.33 & 15.74 & $13.89^{* *}$ & 12.98 & 14.86 & $7.98^{* *}$ & 7.66 & 8.31 & $4.00^{* *}$ & 3.80 & 4.21 \\
\hline \multicolumn{16}{|l|}{ Smoking (Yes as reference) } \\
\hline No & $1.35^{* *}$ & 1.29 & 1.42 & $1.51^{* *}$ & 1.42 & 1.61 & $1.45^{* *}$ & 1.37 & 1.52 & $1.08^{* *}$ & 1.05 & 1.12 & $1.10^{* *}$ & 1.05 & 1.15 \\
\hline \multicolumn{16}{|l|}{ Drinking (Yes as reference) } \\
\hline No & $1.60^{* *}$ & 1.52 & 1.69 & $2.21^{* *}$ & 2.05 & 2.38 & $1.98^{* *}$ & 1.87 & 2.10 & $1.14^{* *}$ & 1.10 & 1.18 & $1.29^{* *}$ & 1.23 & 1.36 \\
\hline \multicolumn{16}{|c|}{ Regular physical activity (Yes as reference) } \\
\hline No & $1.61^{* *}$ & 1.53 & 1.69 & $1.96^{* *}$ & 1.83 & 2.09 & $1.74^{* *}$ & 1.65 & 1.83 & $1.12^{* *}$ & 1.09 & 1.16 & $1.30^{* *}$ & 1.24 & 1.36 \\
\hline \multicolumn{16}{|c|}{ Educational attainment (Illiterate as reference) } \\
\hline Primary school & $0.72^{* *}$ & 0.69 & 0.75 & $0.67^{* *}$ & 0.64 & 0.71 & $0.66^{* *}$ & 0.63 & 0.69 & $0.79 * *$ & 0.76 & 0.81 & $0.69^{* *}$ & 0.66 & 0.72 \\
\hline Junior middle school & $0.55^{* *}$ & 0.52 & 0.58 & $0.52^{* *}$ & 0.48 & 0.55 & $0.49^{* *}$ & 0.47 & 0.52 & $0.57^{* *}$ & 0.55 & 0.59 & $0.55^{* *}$ & 0.52 & 0.58 \\
\hline Senior middle school & $0.46^{* *}$ & 0.43 & 0.49 & $0.43^{* *}$ & 0.40 & 0.48 & $0.41^{* *}$ & 0.38 & 0.45 & $0.48^{* *}$ & 0.46 & 0.51 & $0.48^{* *}$ & 0.45 & 0.51 \\
\hline University/college or above & $0.35^{* *}$ & 0.32 & 0.39 & $0.34^{* *}$ & 0.29 & 0.39 & $0.31^{* *}$ & 0.27 & 0.35 & $0.41^{* *}$ & 0.38 & 0.44 & $0.44^{* *}$ & 0.40 & 0.49 \\
\hline \multicolumn{16}{|c|}{ Household income ranking in local ( $<20 \%$ as reference) } \\
\hline $20 \%-$ & $0.76^{* *}$ & 0.73 & 0.80 & $0.77^{* *}$ & 0.73 & 0.82 & $0.74^{* *}$ & 0.71 & 0.78 & $0.80^{* *}$ & 0.77 & 0.83 & $0.74^{* *}$ & 0.71 & 0.78 \\
\hline $40 \%-$ & $0.69^{* *}$ & 0.66 & 0.73 & $0.72^{* *}$ & 0.67 & 0.76 & $0.67^{* *}$ & 0.64 & 0.71 & $0.75^{* *}$ & 0.72 & 0.78 & $0.65^{* *}$ & 0.62 & 0.68 \\
\hline $60 \%-$ & $0.63^{* *}$ & 0.60 & 0.66 & $0.65^{* *}$ & 0.61 & 0.70 & $0.63^{* *}$ & 0.59 & 0.66 & $0.68^{* *}$ & 0.66 & 0.71 & $0.58^{* *}$ & 0.55 & 0.61 \\
\hline Highest ( $\geq 80 \%)$ & $0.63^{* *}$ & 0.60 & 0.66 & $0.68^{* *}$ & 0.64 & 0.73 & $0.63^{* *}$ & 0.60 & 0.67 & $0.67^{* *}$ & 0.65 & 0.70 & $0.57^{* *}$ & 0.54 & 0.60 \\
\hline \multicolumn{16}{|l|}{ Residency (Urban as reference) } \\
\hline Rural & $0.84^{* *}$ & 0.80 & 0.87 & $0.87^{* *}$ & 0.83 & 0.92 & $0.91^{* *}$ & 0.87 & 0.95 & $0.93^{* *}$ & 0.90 & 0.96 & $0.96^{*}$ & 0.92 & 1.00 \\
\hline \multicolumn{16}{|l|}{ Region (Eastern as reference) } \\
\hline Central & $1.17^{* *}$ & 1.13 & 1.22 & $1.20^{* *}$ & 1.14 & 1.27 & $1.17^{* *}$ & 1.12 & 1.23 & $1.23^{* *}$ & 1.19 & 1.27 & $1.26^{* *}$ & 1.21 & 1.32 \\
\hline Western & $1.29^{* *}$ & 1.24 & 1.34 & $1.29 * *$ & 1.22 & 1.35 & $1.34^{* *}$ & 1.29 & 1.40 & $1.35^{* *}$ & 1.31 & 1.39 & $1.75^{* *}$ & 1.68 & 1.81 \\
\hline \multicolumn{16}{|l|}{ Year (2008 as reference) } \\
\hline 2013 & $1.07^{* *}$ & 1.04 & 1.11 & $0.90^{* *}$ & 0.86 & 0.94 & $0.92^{* *}$ & 0.89 & 0.96 & $1.34^{* *}$ & 1.31 & 1.38 & $0.80^{* *}$ & 0.77 & 0.83 \\
\hline
\end{tabular}

self-care and usual activities decreased. The mixture of changing health problems resulted in a small decline in utility index. However, VAS scores defied the trend, showing a slight increase over time. It is worth noting that the time changes in both indicators were small and failed to reach the threshold of clinical importance in this study. Further studies are needed to verify the contradictory changing trends and their policy and clinical implications. There is evidence that these two indicators measure quite different constructs of HRQoL [68]. VAS scores capture real-time individual ratings, whereas the utility index applies past population preferences to value the current health status. Certain populations (for example women) may have higher than average expectations on health, leading to relatively lower ratings on VAS $[18,19]$.
Age is a significant predictor of HRQoL. Older people are more likely to experience health problems than their younger counterparts as indicated in this study and others $[4,6,18,20,45,69-75]$, resulting in significant lower utility index and VAS scores. The effect sizes of age are the largest among all of the independent variables assessed. This indicates a great challenge to China as its population is ageing rapidly. Previous studies reported consistent lower utility index and VAS scores in women compared with men $[4,6,45,71-73,75-77]$. But interestingly, gender differences in both the utility index and VAS scores were found to be too small to be deemed clinically important in this study and there is no evidence to support a claim of lower utility index in women. In line with in studies conducted elsewhere in China [78-80], this study also provides additional evidence 
Table 5 Multiple linear and Tobit regression analyses on the health utility index and VAS scores

\begin{tabular}{|c|c|c|c|c|c|c|c|c|c|c|c|c|}
\hline \multirow[t]{2}{*}{ Independent variable } & \multicolumn{6}{|c|}{ Utility index } & \multicolumn{6}{|c|}{ VAS scores } \\
\hline & $\beta$ & SE & $p$ & \multicolumn{2}{|l|}{$95 \% \mathrm{Cl}$} & Effect sizes & $\beta$ & SE & $p$ & \multicolumn{2}{|l|}{$95 \% \mathrm{Cl}$} & Effect sizes \\
\hline \multicolumn{13}{|l|}{ Gender (Male as reference) } \\
\hline Female & 0.00 & 0.00 & 0.311 & -0.00 & 0.00 & 0.03 & -0.55 & 0.06 & $<0.001$ & -0.66 & -0.44 & -0.04 \\
\hline \multicolumn{13}{|l|}{ Age ( $<45$ years as reference) } \\
\hline $45 \sim 64$ & -0.11 & 0.00 & $<0.001$ & -0.11 & -0.10 & -1.92 & -7.01 & 0.05 & $<0.001$ & -7.11 & -6.91 & -0.51 \\
\hline $65 \sim$ & -0.21 & 0.00 & $<0.001$ & -0.21 & -0.21 & -3.74 & -13.59 & 0.08 & $<0.001$ & -13.75 & -13.43 & -0.99 \\
\hline \multicolumn{13}{|l|}{ Smoking (Yes as reference) } \\
\hline No & -0.02 & 0.00 & $<0.001$ & -0.02 & -0.01 & -0.28 & -0.18 & 0.06 & 0.006 & -0.30 & -0.05 & -0.01 \\
\hline \multicolumn{13}{|l|}{ Drinking (Yes as reference) } \\
\hline No & -0.02 & 0.00 & $<0.001$ & -0.03 & -0.02 & -0.40 & -0.95 & 0.06 & $<0.001$ & -1.08 & -0.83 & -0.07 \\
\hline \multicolumn{13}{|c|}{ Regular physical activity (Yes as reference) } \\
\hline No & -0.02 & 0.00 & $<0.001$ & -0.03 & -0.02 & -0.43 & -0.50 & 0.06 & $<0.001$ & -0.62 & -0.39 & -0.04 \\
\hline \multicolumn{13}{|c|}{ Educational attainment (Illiterate as reference) } \\
\hline Primary school & 0.03 & 0.00 & $<0.001$ & 0.03 & 0.04 & 0.61 & 2.39 & 0.09 & $<0.001$ & 2.21 & 2.56 & 0.17 \\
\hline Junior middle school & 0.07 & 0.00 & $<0.001$ & 0.06 & 0.07 & 1.16 & 4.46 & 0.09 & $<0.001$ & 4.29 & 4.64 & 0.33 \\
\hline Senior middle school & 0.08 & 0.00 & $<0.001$ & 0.08 & 0.09 & 1.44 & 5.11 & 0.10 & $<0.001$ & 4.91 & 5.31 & 0.37 \\
\hline University/college or above & 0.09 & 0.00 & $<0.001$ & 0.09 & 0.10 & 1.68 & 5.25 & 0.12 & $<0.001$ & 5.02 & 5.48 & 0.38 \\
\hline \multicolumn{13}{|c|}{ Household income ranking in local ( $<20 \%$ as reference) } \\
\hline $20 \%-$ & 0.03 & 0.00 & $<0.001$ & 0.02 & 0.03 & 0.50 & 1.69 & 0.08 & $<0.001$ & 1.54 & 1.85 & 0.12 \\
\hline $40 \%-$ & 0.04 & 0.00 & $<0.001$ & 0.04 & 0.04 & 0.69 & 2.36 & 0.08 & $<0.001$ & 2.21 & 2.51 & 0.17 \\
\hline $60 \%-$ & 0.05 & 0.00 & $<0.001$ & 0.04 & 0.05 & 0.84 & 2.78 & 0.08 & $<0.001$ & 2.63 & 2.93 & 0.20 \\
\hline Highest ( $\geq 80 \%)$ & 0.05 & 0.00 & $<0.001$ & 0.04 & 0.05 & 0.86 & 3.22 & 0.08 & $<0.001$ & 3.07 & 3.37 & 0.23 \\
\hline \multicolumn{13}{|l|}{ Residency (Urban as reference) } \\
\hline Rural & 0.01 & 0.00 & $<0.001$ & 0.01 & 0.02 & 0.26 & 1.65 & 0.05 & $<0.001$ & 1.54 & 1.76 & 0.12 \\
\hline \multicolumn{13}{|l|}{ Region (Eastern as reference) } \\
\hline Central & -0.02 & 0.00 & $<0.001$ & -0.02 & -0.01 & -0.31 & -1.73 & 0.06 & $<0.001$ & -1.84 & -1.62 & -0.13 \\
\hline Western & -0.03 & 0.00 & $<0.001$ & -0.03 & -0.03 & -0.56 & -2.71 & 0.05 & $<0.001$ & -2.82 & -2.61 & -0.20 \\
\hline \multicolumn{13}{|l|}{ Year (2008 as reference) } \\
\hline 2013 & -0.01 & 0.00 & $<0.001$ & -0.01 & -0.01 & -0.20 & 1.61 & 0.05 & $<0.001$ & 1.51 & 1.70 & 0.12 \\
\hline
\end{tabular}

on the associations between behavioral factors (smoking, drinking, and exercise) and HRQoL.

Socioeconomic disparities in HRQoL deserve a serious concern in China. Low socioeconomic status appeared to be associated with lower HRQoL regardless of which indicator was applied in this study. Those who were wealthier and had higher educational attainments had higher scores in both utility and VAS scores. In line with regional disparities in socioeconomic development in China, higher scores in utility and VAS scores were also found for those who resided in the more developed eastern region. These results are consistent with findings of other studies [4, 6, 18-20, 45, 69-72, 74, 81]. Between 2008 and 2013, China's wealth increased exponentially, which was accompanied by dramatic improvement of people's living standards. Life expectancy increased from 74.8 to 76.3 years over the period of time [82]. Unfortunately, this development had not translated into a higher HRQoL as indicated in the crude utility index and VAS scores results.

China experienced rapid urbanisation process over the past few decades. But rural residents still comprised $46.27 \%$ of the total population in 2013 [82]. This study adopted the value sets derived from a sample inclusive of rural residents. It showed that rural residents reported more health problems compared with their urban counterparts, but they had higher scores in the utility index and VAS scores after controlling for other factors. Clearly, urbanisation is unlikely to offer a silver bullet to improving the HRQoL of Chinese people. Future studies should explore whether rural residents in China hold different perceptions and values on health problems. Many young people in rural and undeveloped regions chose to leave their villages, hoping to live a better life 
[22, 24, 83-85]. This may have significant implications on both individual ratings and population preferences on health.

\section{Limitations}

There are several limitations in this study. Although the study sample is large and representative of the nation, we do not have control over the choice of data and questions definitions $[18,19]$. The two rounds of cross-sectional surveys were not undertaken using the same pool of participants $[65,86]$, which prevents us from drawing casual conclusions. In addition, the EQ-5D-3 L used in the NHSS has more significant ceiling effects than the EQ-5D-5 L. However, the comparison of the two large national cross-sectional surveys provides valuable indications on the overall changing trends of HRQoL, which is important to inform future studies.

\section{Conclusions}

The changing trend (decrease) of the utility index is contradictory to that (increase) of the VAS in China over the period between 2008 and 2013, although neither is deemed clinically important. Such a change (or lack of) cannot be fully explained by the changing demographic and socioeconomic status in China. Further studies are warranted to explore the underlining reasons, in particular the role of health reforms [64]. Meanwhile, it is important to note that socioeconomic and regional disparities in HRQoL are increasing in China. The rapid socioeconomic transition and demographic changes in China may also have some potential impacts on public preferences on health status.

\section{Abbreviations \\ AD: Anxiety/depression; ANOVA: Analysis of Variance; AOR: Adjusted Odds Ratio; EQ-5D: European Quality of Life Five Dimensions; EQ-5D-3 L: 3-level EQ-5D; EQ-5D-5 L: 5-level EQ-5D; HRQoL: Health-Related Quality of Life; MO: Mobility; NHSS: National Health Services Surveys; PD: Pain/discomfort; QALYs: Quality-Adjusted Life Years; SC: Self-care; SD: Standard Deviation; TTO: Time Trade-Off; UA: Usual Activities; VAS: Visual Analogue Scale}

\section{Acknowledgements}

The authors thank the Centre for Health Statistics and Information, National Health and Family Planning Commission of China for providing data support. We would like to thank all of the participants in the NHSS, and the reviewers for their insightful comments that help improve the manuscript.

\section{Funding}

The study was funded by the National Natural Science Foundation of China (grant numbers 71373183 and 71603188), the Humanity and Social Science Youth Foundation from the Ministry of Education of China (grant numbers 16YJCZH137) and the Fundamental Research Funds for the Central Universities (grant numbers 2018QN051).

\section{Availability of data and materials}

The data that support the findings of this study are available from the Centre for Health Statistics and Information, National Health Commission. But restrictions apply to the availability of these data, which were used under the license for the current study. They are not publicly available.

\section{Authors' contributions}

QY, YZ and LX contributed to the study design, data analyses, data interpretation, and drafting of the manuscript. CL contributed to data analyses, interpretation of results and writing of the manuscript. All authors have read and approved the final version of the manuscript.

\section{Ethics approval and consent to participate}

The NHSS obtained ethics approval (license number: 2008 (18) and 2013 (65)) from the institutional review board of the Chinese National Bureau of Statistics. All procedures performed in the studies involving human participants were in accordance with the ethical standards of the institutional and/or national research committee and with the 1964 Helsinki declaration and its later amendments or comparable ethical standards. The interviewers explained the purpose of the survey before obtaining oral informed consent from the individual participants.

Consent for publication

Not applicable.

\section{Competing interests}

The authors declare that they have no competing interests.

\section{Publisher's Note}

Springer Nature remains neutral with regard to jurisdictional claims in published maps and institutional affiliations.

\section{Author details}

${ }^{1}$ School of Political Science and Public Administration, Institute of Health Research, Wuhan University, Wuhan, Hubei, China. ${ }^{2}$ School of Psychology and Public Health, La Trobe University, Melbourne, VIC, Australia. ${ }^{3}$ Center for Health Statistics and Information, National Health Commission, Beijing, China. ${ }^{4}$ Health Human Resources Development Center, National Health

Commission, Beijing, China.

Received: 24 September 2018 Accepted: 21 February 2019

Published online: 11 March 2019

\section{References}

1. Karimi M, Brazier J. Health, health-related quality of life, and quality of life: what is the difference? Pharmacoeconomics. 2016;34:645-9.

2. Yin S, Njai R, Barker L, Siegel PZ, Liao Y. Summarizing health-related quality of life (HRQOL): development and testing of a one-factor model. Popul Health Metrics. 2016;14:22.

3. Rabin R, de Charro F. EQ-5D: a measure of health status from the EuroQol group. Ann Med. 2001;33:337-43.

4. Zhang Y, Zhou Z, Gao J, Wang D, Zhang Q, Zhou Z, Su M, Li D. Healthrelated quality of life and its influencing factors for patients with hypertension: evidence from the urban and rural areas of Shaanxi Province, China. BMC Health Serv Res. 2016;16:277.

5. Scalone L, Cortesi PA, CIAMpICHINI R, Cesana G, Mantovani LG. Health related quality of life norm data of the Italian general population: results using the EQ-5D-3L and EQ-5D-5L instruments. Epidemiol Biostat Public Health. 2015;12:e11457-1-e11457-15.

6. Shiroiwa T, Fukuda T, Ikeda S, Igarashi A, Noto S, Saito S, Shimozuma K. Japanese population norms for preference-based measures: EQ-5D-3L, EQ5D-5L, and SF-6D. Qual Life Res. 2016;25:707-19.

7. Brazier J, Ratcliffe J, Saloman J, Tsuchiya A. Measuring and valuing health benefits for economic evaluation. Oxford: Oxford University Press; 2017.

8. Payakachat N, Ali MM, Tilford JM. Can the EQ-5D detect meaningful change? A systematic review. Pharmacoeconomics. 2015;33:1137-54.

9. Hjelmgren J, Berggren F, Andersson F. Health economic guidelines-similarities, differences and some implications. Value Health. 2001:4:225-50.

10. McCaffrey N, Kaambwa B, Currow DC, Ratcliffe J. Health-related quality of life measured using the EQ-5D-5L: south Australian population norms. Health Qual Life Outcomes. 2016;14:133.

11. Merlo G, Page K, Ratcliffe J, Halton K, Graves N. Bridging the gap: exploring the barriers to using economic evidence in healthcare decision making and strategies for improving uptake. Appl Health Econ Health Policy. 2015;13:303-9. 
12. Romero M, Vivas-Consuelo D, Alvis-Guzman N. Is health related quality of life (HRQoL) a valid indicator for health systems evaluation? SpringerPlus. 2013;2:664.

13. Sullivan SM, Wells $G$, Coyle D. What guidance are economists given on how to present economic evaluations for policymakers? A systematic review. Value Health. 2015;18:915-24.

14. Devlin NJ, Brooks R. EQ-5D and the EuroQol group: past, present and future. Appl Health Econ Health Policy. 2017;15:127-37.

15. Liu GG, Wu H, Li M, Gao C, Luo N. Chinese time trade-off values for EQ-5D health states. Value Health. 2014;17:597-604.

16. Wang $\mathrm{H}$, Kindig DA, Mullahy J. Variation in Chinese population health related quality of life: results from a EuroQol study in Beijing, China. Qual Life Res. 2005;14:119-32.

17. Wang H-M, Patrick DL, Edwards TC, Skalicky AM, Zeng H-Y, Gu W-W. Validation of the EQ-5D in a general population sample in urban China. Qual Life Res. 2012;21:155-60.

18. Sun $S$, Chen J, Johannesson M, Kind P, Xu L, Zhang Y, Burström $K$. Population health status in China: EQ-5D results, by age, sex and socioeconomic status, from the National Health Services Survey 2008. Qual Life Res. 2011;20:309-20

19. Sun $S$, Chen J, Johannesson $M$, Kind $P, X u$ L, Zhang $Y$, Burström $K$ Regional differences in health status in China: population health-related quality of life results from the National Health Services Survey 2008. Health Place. 2011;17:671-80.

20. Huang W, Yu H, Liu C, Liu G, Wu Q, Zhou J, Zhang X, Zhao X, Shi L, Xu X. Assessing health-related quality of life of chinese adults in Heilongjiang using EQ-5D-3L. Int J Environ Res Public Health. 2017;14:224.

21. Sun X, Lucas H, Meng Q, Zhang Y. Associations between living arrangements and health-related quality of life of urban elderly people: a study from China. Qual Life Res. 2011;20:359-69.

22. Liang $Y$, Wu W. Exploratory analysis of health-related quality of life among the empty-nest elderly in rural China: an empirical study in three economically developed cities in eastern China. Health Qual Life Outcomes. 2014;12:59.

23. Li H, Wei X, Ma A, Chung RY. Inequalities in health status among rural residents: EQ-5D findings from household survey China. Int J Equity Health. 2014;13:41.

24. Lei $\mathrm{P}, \mathrm{Xu}$ L, Nwaru B, Long Q, Wu Z. Social networks and health-related quality of life among Chinese old adults in urban areas: results from 4th National Household Health Survey. Public Health. 2016;131:27-39.

25. Zhang T, Shi W, Huang Z, Gao D, Guo Z, Liu J, Chongsuvivatwong V. Influence of culture, residential segregation and socioeconomic development on rural elderly health-related quality of life in Guangxi, China. Health Qual Life Outcomes. 2016;14:98.

26. Zhang L, Guo XL, Zhang JY, Chen X, Zhou CC, Ge DD, Qian YY. Healthrelated quality of life among adults with and without hypertension: a population-based survey using EQ-5D in Shandong, China. Sci Rep. 2017;7:14960

27. Lin $K$, Yang $X$, Yin G, Lin S. Diabetes self-care activities and health-related quality-of-life of individuals with type 1 diabetes mellitus in Shantou, China. J Int Med Res. 2016:44:147-56.

28. Lee CF, Ng R, Luo N, Wong NS, Yap YS, Lo SK, Chia WK, Yee A, Krishna L, Wong $\mathrm{C}$. The English and Chinese versions of the five-level EuroQoL Group's five-dimension questionnaire (EQ-5D) were valid and reliable and provided comparable scores in Asian breast cancer patients. Support Care Cancer. 2013;21:201-9.

29. Wu J, Han Y, Xu J, Lu Y, Cong H, Zheng J, Sun H. Chronic stable angina is associated with lower health-related quality of life: evidence from Chinese patients. PLoS One. 2014;9:e97294.

30. Gao L, Xia L, Pan S-Q, Xiong T, Li S-C. Reliability and validity of QOLIE-10 in measuring health-related quality of life (HRQoL) in Chinese epilepsy patients. Epilepsy Res. 2014;108:565-75.

31. Sun J, Zhao Y, Yang R, Guan T, lorio A. The demographics, treatment characteristics and quality of life of adult people with haemophilia in China-results from the HERO study. Haemophilia. 2017;23:89-97.

32. Qiu Y, Zhao K, Li X, Shi L, Guo W, Qi X, Sui B, Zhou R. Health economic evaluation of a 23 value pneumococcal polysaccharide vaccination pilot programme among elderly chronic obstructive pulmonary disease patients in China. Zhonghua Yu Fang Yi Xue Za Zhi. 2016;50:1074-8.

33. Fang $H$, Farooq U, Wang D, Yu F, Younus Ml, Guo X. Reliability and validity of the EQ-5D-3L for Kashin-Beck disease in China. SpringerPlus. 2016;5:1924.
34. Wang S-I WB, Leng L, Bucala R, Lu LJ. Validity of LupusQoL-China for the assessment of health related quality of life in Chinese patients with systemic lupus erythematosus. PLoS One. 2013;8:e63795.

35. Janssen MF, Szende A, Cabases J, Ramos-Goni JM, Vilagut G, Konig HH. Population norms for the EQ-5D-3L: a cross-country analysis of population surveys for 20 countries. Eur J Health Econ. 2018 (online 14 February):1-12.

36. Bailey $H$, Kind P. Preliminary findings of an investigation into the relationship between national culture and EQ-5D value sets. Qual Life Res. 2010;19:1145-54.

37. Knies S, Evers SM, Candel MJ, Severens JL, Ament AJ. Utilities of the EQ-5D: transferable or not? Pharmacoeconomics. 2009;27:767-79.

38. Xu Y, Zhou Z, Li Y, Yang J, Guo X, Gao J, Je Y, Chen G. Exploring the nonlinear relationship between body mass index and health-related quality of life among adults: a cross-sectional study in Shaanxi Province, China. Health Qual Life Outcomes. 2015:13:153.

39. Su M, Zhou Z, Si Y, Wei X, Xu Y, Fan X, Chen G. Comparing the effects of China's three basic health insurance schemes on the equity of healthrelated quality of life: using the method of coarsened exact matching. Health Qual Life Outcomes. 2018:16:41.

40. Zhou Z, Zhou Z, Li D, Wang D, Shi C, Chen C, Fang Y, Gao J, Chen G. Analyzing the health-related quality of life of urban and rural residents in Shaanxi: estimation based on the EQ-5D value sets. Chinese Health Economics. 2015;34:13-6.

41. Wu CM, Gong YH, Wu J, Zhang SC, Yin XX, Dong XX, Li WZ, Cao SY, Mkandawire N, Lu ZX. Chinese version of the EQ-5D preference weights: applicability in a Chinese general population. PLoS One. 2016;11:e0164334

42. Wu H, Liu G, Guan H. Analysis on influential factors of health utility evaluation. China Pharmacy. 2016;27:1450-3.

43. Zhuo L, Xu L, Ye JT, Sun S, Zhang YG, Burstrom K, Chen JY. Time trade-off value set for EQ-5D-3L based on a nationally representative Chinese population survey. Value Health. 2018;21:1330-7.

44. Oppe M, Devlin NJ, Szende A. EQ-5D value sets: inventory, comparative review and user guide. Berlin: Springer; 2007.

45. Szende A, Janssen B, Cabases J. Self-reported population health: an international perspective based on EQ-5D. Dordrecht (The Netherlands): Springer; 2014.

46. Szende A, Williams A. Measuring self-reported population health: an international perspective based on EQ-5D. SpringMed publishing; 2004.

47. Qian J, Cai M, Gao J, Tang S, Xu L, Critchley JA. Trends in smoking and quitting in China from 1993 to 2003: National Health Service Survey data. Bull World Health Organ. 2010(88):769-76.

48. Li J, Wen Z, Cai A, Tian F, Zhang L, Luo X, Deng L, He J, Yang Y, Chen W. Real-world cost-effectiveness of infliximab for moderate-to-severe rheumatoid arthritis in a medium-sized city of China. J Comp Eff Res. 2017;6:205-18.

49. Meng Q, Xu L, Zhang Y, Qian J, Cai M, Xin Y, Gao J, Xu K, Boerma JT, Barber SL. Trends in access to health services and financial protection in China between 2003 and 2011: a cross-sectional study. Lancet. 2012:379:805-14

50. Center for Health Statistics and Information MoHC. An Analysis Report of National Health Services Survey in China, 2013, vol. 2015. Beijing: Center for health statistics and information, Ministry of Health China.

51. Qian Y, Zhou Z, Je Y, Gao J, Wang Y, Yang X, Xu Y, Li Y. An economy-related equity analysis of health service utilization by women in economically underdeveloped regions of western China. Int J Equity Health. 2017:16:186.

52. Sun H, Zhang Q, Luo X, Quan H, Zhang F, Liu C, Liu M. Changes of adult population health status in China from 2003 to 2008. PLoS One. 2011:6:e28411.

53. Center for Health Statistics and Information MoHC. Analysis Report of National Health Services Survey in China, 2008, vol. 2009. Beijing: Center for health statistics and information, Ministry of Health China.

54. Zhuo L, Wang G, Ye J, Gong J, Liu Y, Xu L, Wang X. Exploring EQ-5D value set with standard gamble method. J Zhengzhou Univ (Med Sci). 2018;53:88-93.

55. Durch JS, Bailey LA, Stoto MA. Improving health in the community: a role for performance monitoring. Washington, D.C. US: National Academies Press; 1997.

56. Institute of Medicine. Committee on Assuring the Health of the Public in the 21st Century. The Future of the Public's Health in the 21st Century. Washington, D.C. US: National Academy Press; 2003.

57. Glouberman S, Millar J. Evolution of the determinants of health, health policy, and health information systems in Canada. Am J Public Health 2003;93:388-92

58. Bambra C, Gibson M, Sowden A, Wright K, Whitehead M, Petticrew M. Tackling the wider social determinants of health and health inequalities: 
evidence from systematic reviews. J Epidemiol Community Health. 2010;64: 284-91.

59. Dahlgren G, Whitehead M. Policies and strategies to promote social equity in health. Institute for future studies: Stockholm; 1991.

60. Evans RG, Stoddart GL. Producing health, consuming health care. Why are some people healthy and others not: 1994. p. 27-64.

61. Marmot M. Social determinants of health inequalities. Lancet. 2005;365:1099-104.

62. People H: 2020.(2011). Healthy people 2011, 2020.

63. Feng $\mathrm{XL}, \mathrm{Xu} \mathrm{L}$, Guo Y, Ronsmans C. Socioeconomic inequalities in hospital births in China between 1988 and 2008. Bull World Health Organ. 2011;89:432-41.

64. Yip WC, Hsiao WC, Chen W, Hu S, Ma J, Maynard A. Early appraisal of China's huge and complex health-care reforms. Lancet. 2012;379:833-42.

65. Zhou Z, Su Y, Gao J, Xu L, Zhang Y. New estimates of elasticity of demand for healthcare in rural China. Health Policy. 2011;103:255-65.

66. Cohen J. Statistical power analysis. Curr Dir Psychol Sci. 1992;1:98-101.

67. Norman GR, Sloan JA, Wyrwich KW. Interpretation of changes in healthrelated quality of life: the remarkable universality of half a standard deviation. Med Care. 2003;41:582-92.

68. Ebrahim S, Parshuram C. Comparison of utility scores from the visual analog scale and health utilities index 3 in children following pediatric intensive care unit admission. J Child Health Care. 2015;19:53-62.

69. Luo N, Johnson JA, Shaw JW, Feeny D, Coons SJ. Self-reported health status of the general adult US population as assessed by the EQ-5D and health utilities index. Med Care. 2005;43:1078-86.

70. Sørensen J, Davidsen M, Gudex C, Pedersen KM, Brønnum-Hansen H. Danish EQ-5D population norms. Scand J Public Health. 2009:37:467-74.

71. Abdin E, Subramaniam M, Vaingankar JA, Luo N, Chong SA. Measuring health-related quality of life among adults in Singapore: population norms for the EQ-5D. Qual Life Res. 2013;22:2983-91.

72. Clemens S, Begum N, Harper C, Whitty JA, Scuffham PA. A comparison of EQ-5D-3L population norms in Queensland, Australia, estimated using utility value sets from Australia, the UK and USA. Qual Life Res. 2014;23:2375-81.

73. Ferreira LN, Ferreira PL, Pereira LN, Oppe M. EQ-5D Portuguese population norms. Qual Life Res. 2014;23:425-30.

74. Kularatna S, Whitty JA, Johnson NW, Jayasinghe R, Scuffham PA. EQ-5D-3L derived population norms for health related quality of life in Sri Lanka. PLoS One. 2014;9:e108434.

75. Kind P, Dolan P, Gudex C, Williams A. Variations in population health status: results from a United Kingdom national questionnaire survey. BMJ. 1998;316:736-41.

76. Perneger TV, Combescure C, Courvoisier DS. General population reference values for the French version of the EuroQol EQ-5D health utility instrument. Value Health. 2010;13:631-5.

77. Golicki D, Niewada M. General population reference values for 3-level EQ-5D (EQ-5D-3L) questionnaire in Poland. Pol Arch Med Wewn. 2015;125:18-26.

78. Tan Z, Liang Y, Liu S, Cao W, Tu H, Guo L, Xu Y. Health-related quality of life as measured with EQ-5D among populations with and without specific chronic conditions: a population-based survey in Shaanxi Province, China. PLoS One. 2013;8:e65958.

79. Cui J, Tang J, Qin J, Zhang C, Chen Y. Self-evaluated health condition and its influencing factors of residents in Hami area. Chinese General Pract. 2015;8:023.

80. Wang $\mathrm{X}$, Guo H, Chen J. An empirical study on self-rated health status and reporting behaviors of rural residents in China. Chinese J Health Policy. 2016;9:68-74.

81. Abdin E, Subramaniam M, Vaingankar JA, Luo N, Chong SA. Population norms for the EQ-5D index scores using Singapore preference weights. Qual Life Res. 2015;24:1545-53.

82. Statistical Communiqué of the People's Republic of China on the National Economic and Social Development [http://www.stats.gov.cn/tisj/ndsj/2018/ indexch.htm ]. Accessed 8 Feb 2019.

83. Li H, Tracy MB. Family support, financial needs, and health care needs of rural elderly in China: a field study. J Cross-Cultural Gerontol. 1999;14:357-71.

84. Liang $Y$, Y $Y$ Y, Sun $Q$. The impact of migration on fertility under China's underlying restrictions: a comparative study between permanent and temporary migrants. Soc Indic Res. 2014;116:307-26.

85. Zhaiping H. Socioeconomic status and social support network of the rural elderly and their physical and mental health. Soc Sci In China. 2002;3:11.
86. Zhou Z, Su Y, Gao J, Campbell B, Zhu Z, Xu L, Zhang Y. Assessing equity of healthcare utilization in rural China: results from nationally representative surveys from 1993 to 2008. Int J Equity Health. 2013;12:34.

\section{Ready to submit your research? Choose BMC and benefit from:}

- fast, convenient online submission

- thorough peer review by experienced researchers in your field

- rapid publication on acceptance

- support for research data, including large and complex data types

- gold Open Access which fosters wider collaboration and increased citations

- maximum visibility for your research: over $100 \mathrm{M}$ website views per year

At BMC, research is always in progress.

Learn more biomedcentral.com/submissions 